\title{
Full-Field Simulation for Sonic Boom Cutoff Phenomena*
}

\author{
Rei YAMASHITA and Kojiro SUZUKI \\ Department of Advanced Energy, The University of Tokyo, Chiba 277-8561, Japan
}

\begin{abstract}
This study describes an application of full-field, direct simulation in the entire domain involving the near-field around an aircraft, the far-field toward the ground, and the caustic region as an approach to investigate sonic boom cutoff phenomena. A flow field around an axi-symmetric paraboloid has been analyzed by solving three-dimensional (3D) Euler equations with a gravity term for considering atmospheric stratification with altitude. A solution-adapted grid is constructed to align the grid lines with front and rear shock-wave surfaces. The flight is assumed to have a speed of Mach 1.1 at an altitude of $10 \mathrm{~km}$. This study presents the world's first successful numerical simulation for a 3D structure of cutoff phenomena. The results show that the incoming wave generated from the paraboloid is divided into an outgoing wave in the upward direction and an evanescent wave in the downward direction at the cutoff altitude by the diffraction effect. In the cutoff region, the sonic boom reaches the peak intensity by focusing the rays. The evanescent wave under the cutoff region is reduced rapidly. Moreover, the shape of the cutoff surface is obtained for sonic and lateral cutoffs.
\end{abstract}

Key Words: $\quad$ Sonic Boom, Cutoff, Focused Sonic Boom, CFD, Full-Field Simulation

\section{Nomenclature}

$D$ : maximum diameter of body

$E, F, G$ : flux vector at $x, y$, and $z$ coordinates

$E_{t}$ : total energy

$g$ : acceleration of gravity

$h$ : altitude

$L$ : length of body

$p$ : pressure

$Q$ : vector of conservative variables

$r$ : radial coordinate

$R$ : gas constant

$S(x)$ : sectional area at $x$ coordinate

$S_{C}$ : correction term

$S_{G}:$ gravity term

$t$ : time

$T$ : temperature

$u, v, w$ : velocity in $x, y$, and $z$ coordinates

$u_{\text {ref }}$ : flight velocity

$x$ : streamwise coordinate

$y$ : vertical coordinate

$z$ : horizontal coordinate

$\beta$ : atmospheric temperature lapse rate

$\Delta p$ : pressure rise

$\Delta t$ : relative time

$\theta$ : rotational coordinate

Subscripts

$\rho$ : density

0: reference value at ground

max: maximum value

$\infty$ : international standard atmosphere

(C) 2015 The Japan Society for Aeronautical and Space Sciences *Part of this paper was presented at the 2013 Asia-Pacific International Symposium on Aerospace Technology, November 20-22, Takamatsu, Japan. Received 11 September 2014; final revision received 26 January 2015; accepted for publication 27 July 2015.

\section{Introduction}

To avoid the sonic boom problem, a supersonic aircraft flying at a relatively low supersonic speed of Mach 1.2 or less may be possible in the future. ${ }^{1)}$ This speed is preferential because the shock wave generated by low supersonic flight is diffracted by an increase in atmospheric temperature, and the shock wave does not reach the ground. This phenomenon is known as sonic boom cutoff. When cutoff phenomena occur below an aircraft, it may be allowed to make a supersonic flight overland.

However, even if a supersonic aircraft flies at a low supersonic speed, the flight conditions must be determined by considering the influence of sonic boom for the following two reasons: First, cutoff is a type of sonic boom formed by focusing the rays, which indicate the propagation paths of the shock waves, as shown in Fig. 1. The sonic boom strength in the cutoff region becomes approximately two to three times larger than that without the focusing of rays. Therefore, it is imperative that cutoff phenomena are avoided near the ground. Second, because an evanescent wave rather than a shock wave propagates beneath the cutoff region, the flight altitude must be decided by considering the evanescent wave. Thus, clarifying the detailed mechanism of cutoff phenomena is important to decide the appropriate flight conditions.

Thus far, the nature of focused sonic boom has not been well clarified because of its complexity and nonlinearity; therefore, further investigation is necessary. Recently, the focused sonic boom due to transonic acceleration, maneuver, and cutoff was evaluated via two flight tests known as the Superboom Caustic Analysis and Measurement Project $(\mathrm{SCAMP})^{2)}$ and the Farfield Investigation of No Boom Threshold $(\text { FaINT) })^{3)}$ at NASA. In addition, focused sonic boom has been predicted by various methods that solve simplified equations such as the nonlinear progressive-wave equation, ${ }^{4)}$ nonlinear Tricomi equation, ${ }^{5)}$ and lossy nonlinear 


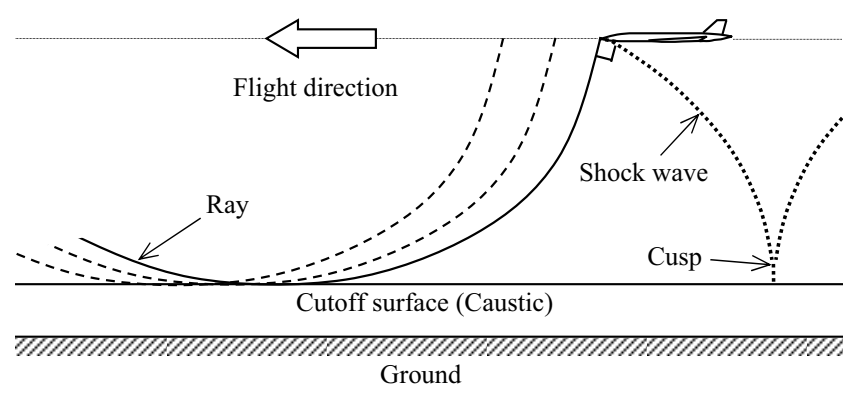

(a) Sonic cutoff

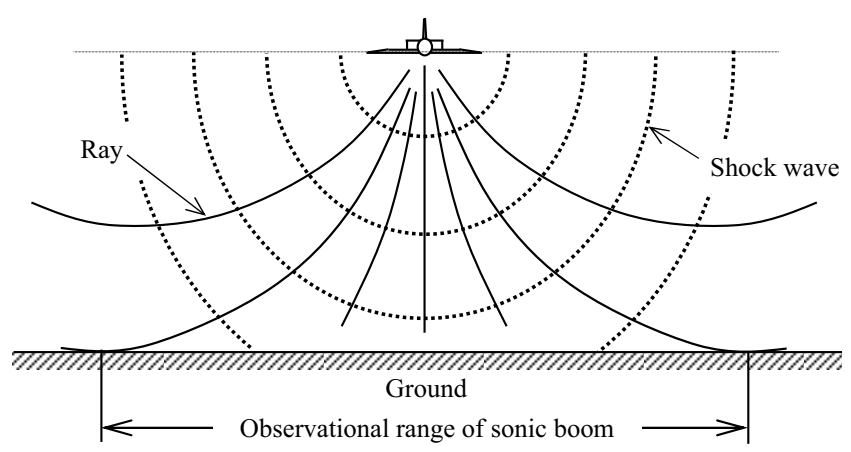

(b) Lateral cutoff

Fig. 1. Sonic boom cutoff phenomena.

Tricomi equation. ${ }^{6}$ However, in this regard, predictions have been made mainly for transonic accelerations and maneuvers, but not for cutoffs. Furthermore, these prediction methods have been applied only to the surrounding area of the caustic region, which indicated the envelope surface of the rays. One reason for this application is that the latest prediction method based on the lossy nonlinear Tricomi equation does not consider two important effects: geometrical spreading of the shock wave with increasing distance from the generation source of the wave and atmospheric stratification with altitude.

In this study, we focused on full-field simulation as a new approach to investigate the cutoff phenomena included in a focused sonic boom. Full-field simulation, which is direct simulation in the entire domain including the near-field around the aircraft, the far-field down to the ground, and the caustic region, is based on computational fluid dynamics (CFD) analysis. Therefore, various effects such as nonlinearity, geometrical spreading, atmospheric stratification, and diffraction can be considered by modifying the governing equations. For the case without the focusing of rays, the usefulness of full-field simulation has been previously confirmed by a comparison of two results as follows ${ }^{7,8)}$ : The first was the result of the waveform parameter method, ${ }^{9)}$ which was a representative evaluation method for sonic boom. The second was the result of the D-SEND\#1 flight test ${ }^{10)}$ conducted by the Japan Aerospace Exploration Agency (JAXA). Full-field simulation is expected to be a powerful tool for investigating the nature of focused sonic boom in the cutoff region because the effects of geometrical spreading and atmospheric stratification can be considered. The objectives of this study are to assess the usefulness of full-field

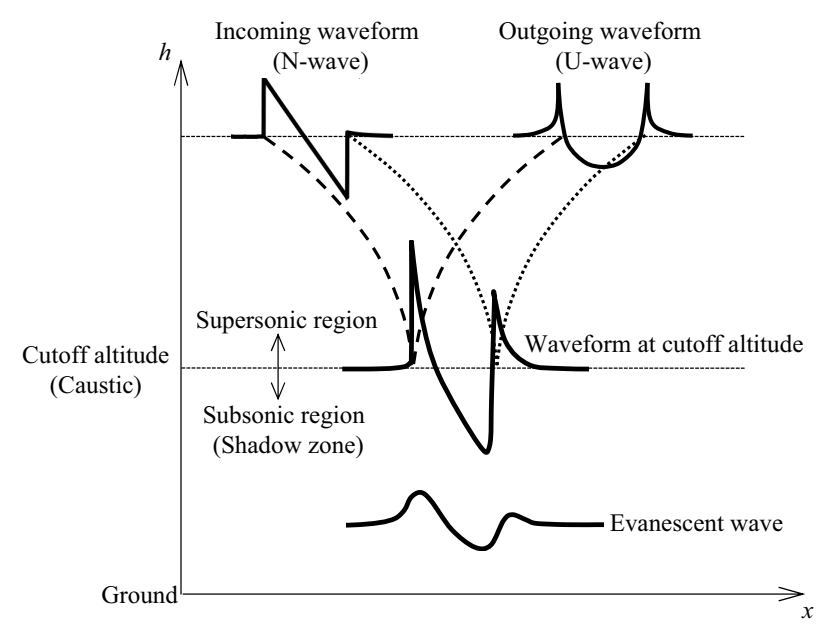

Fig. 2. Schematic of cutoff phenomena.

simulation applied to the cutoff region and investigate the detailed mechanism of cutoff phenomena, such as the characteristics of sonic boom propagation, the focusing strength, and the shape of the cutoff surface.

\section{Cutoff Phenomena}

Cutoff phenomena are divided into two categories: sonic and lateral cutoffs (Fig. 1). The ray that is vertical to the shock wave is the propagation path of the wave. The atmospheric temperature varies with altitude; therefore, the ray is refracted, and the gradient of the ray varies with altitude, as depicted in Fig. 1. As a result, sonic cutoff occurs below the aircraft at a high altitude and a relatively low supersonic Mach number flight, and the shock wave does not reach the ground. When sonic cutoff occurs, several rays overlap in the vicinity of the cutoff altitude, as shown in Fig. 1(a). Then, a cusp of the shock wave is formed in the cutoff region, and sonic boom intensity increases. The shock wave propagating obliquely downward is also refracted by variation in the atmospheric temperature with altitude, which results in lateral cutoff (Fig. 1(b)). The altitude of lateral cutoff is higher than that of sonic cutoff. Thus, lateral cutoff limits the observational range of sonic boom at the ground level normal to the flight direction.

Figure 2 shows a schematic of the cutoff phenomena. ${ }^{5)}$ The caustic and shadow zones indicate the envelope surface of the rays and the area beneath the caustic region, respectively. The incoming wave generated from the body propagates in the downward direction. The incoming wave is divided into two waves by the diffraction effect. One is an outgoing wave in the upward direction, and the other is an evanescent wave in the downward direction. If the incoming waveform is an $\mathrm{N}$-wave, the outgoing waveform assumes a $\mathrm{U}$ shape (U-wave). Considering sonic cutoff, the altitude of the caustic region is equal to the cutoff altitude, where the local Mach number is 1 . Therefore, the upper area of the caustic region is aerodynamically a supersonic region, and the lower area (shadow zone) is a subsonic region; that is, the flow field around the cutoff region is in a transonic flow re- 
gime. The full-field simulation can consider geometrical spreading and atmospheric stratification in addition to nonlinearity and diffraction, and is expected to analyze the cutoff phenomena.

\section{Computational Method}

\subsection{Computational conditions}

A simple computational model is suitable not only for the simplicity of the analysis, but also for reducing the computational cost. The computational model is an axi-symmetric paraboloid given by Eq. (1).

$$
S(x)=4 \pi D^{2}\left(\frac{x}{L}\right)^{2}\left(1-\frac{x}{L}\right)^{2} \quad\left(0 \leq \frac{x}{L} \leq 1\right)
$$

The length of the body is $L=100 \mathrm{~m}$, and its maximum diameter is $D=10 \mathrm{~m}$. The flight is assumed to have a speed of Mach 1.1 at an altitude of $10 \mathrm{~km}$. The computational domain is set from $r / L=0$ to 110 , where the extended region is set from $r / L=100$ to 110 to prevent the influence of the boundary condition at the maximum value of $r$. The results from $r / L=0$ to 100 were investigated.

\subsection{Atmospheric model and governing equations}

To simulate the cutoff phenomena caused by variation in the atmospheric properties with altitude, the atmospheric stratification must be considered. Here, the atmospheric model was set as the ISO International Standard Atmosphere (ISA). ${ }^{11)}$ In ISA, the atmospheric temperature is defined as Eqs. (2) and (3), where $T_{0}=288.15 \mathrm{~K}$ and $\beta=6.5 \mathrm{~K} / \mathrm{km}$.

$$
\begin{gathered}
T_{\infty}=T_{0}-\beta h \quad(0 \leq h \leq 11 \mathrm{~km}) \\
T_{\infty}=\mathrm{const} \quad(11 \leq h \leq 20 \mathrm{~km})
\end{gathered}
$$

Moreover, to calculate the analytical solutions of atmospheric pressure and density, the equation of the state of ideal gas and the hydrostatic equation are defined as Eqs. (4) and (5), respectively, where $p_{0}=101.325 \mathrm{kPa}$.

$$
\begin{aligned}
& p_{\infty}=\rho_{\infty} R T_{\infty} \\
& \frac{\mathrm{d} p_{\infty}}{\mathrm{d} h}=-g \rho_{\infty}
\end{aligned}
$$

Figure 3(a) shows the atmospheric temperature determined by Eqs. (2) and (3). Figure 3(b) shows the analytical solution of atmospheric pressure obtained by Eqs. (4) and (5).

The governing equations are three-dimensional (3D) Euler equations given by Eq. (6), including the gravity term $S_{G}$ for considering atmospheric stratification.

$$
\frac{\partial Q}{\partial t}+\frac{\partial E}{\partial x}+\frac{\partial F}{\partial y}+\frac{\partial G}{\partial z}=S_{G}
$$

$$
Q=\left[\begin{array}{c}
\rho \\
\rho u \\
\rho v \\
\rho w \\
E_{t}
\end{array}\right], \quad E=\left[\begin{array}{c}
\rho u \\
\rho u^{2}+p \\
\rho u v \\
\rho u w \\
\left(E_{t}+p\right) u
\end{array}\right], \quad F=\left[\begin{array}{c}
\rho v \\
\rho u v \\
\rho v^{2}+p \\
\rho v w \\
\left(E_{t}+p\right) v
\end{array}\right]
$$

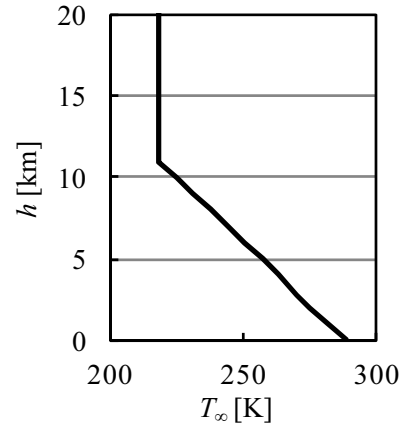

(a) Temperature

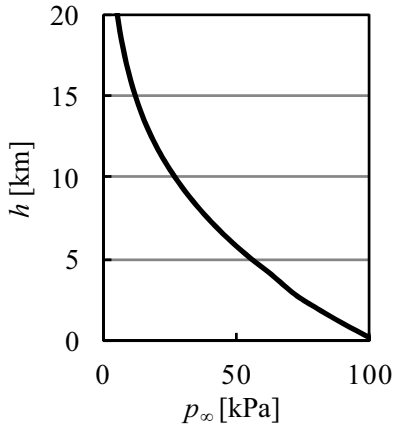

(b) Pressure
Fig. 3. International standard atmosphere. ${ }^{11)}$

$$
G=\left[\begin{array}{c}
\rho w \\
\rho u w \\
\rho v w \\
\rho w^{2}+p \\
\left(E_{t}+p\right) w
\end{array}\right], \quad S_{G}=\left[\begin{array}{c}
0 \\
0 \\
\rho g \\
0 \\
\rho g v
\end{array}\right]
$$

To reduce the computational cost in this simulation, the effects of viscosity and molecular relaxation were not considered.

\subsection{Computational approach}

In the ISA model of stratified atmosphere, the pressure was changed in the vertical direction. This change was assumed as a discontinuity in the Riemann solver; that is, if the state of hydrostatic equilibrium is not strictly maintained, nonphysical waves are generated in the stratified atmosphere. Generally, the Riemann solver cannot distinguish between changes by the stratified atmosphere and fluctuation by the waves in a compressible fluid. For this reason, the generation of nonphysical waves by the stratified atmosphere must not occur. To avoid this generation, an approach that was used in the full-field simulation is as follows.

In the simulation, the governing equations must be discretized to use the computational grid. From the Taylor series expansion of Eq. (5),

$$
\frac{\Delta p_{\infty}}{\Delta h}=-g \rho_{\infty}+O\left(\Delta h^{2}\right)
$$

where the second term in the right-hand side is a discretization error. If the error is ignored, the state of hydrostatic equilibrium cannot be strictly maintained. When the governing equations are discretized, specifically as that in Eq. (6), the discretization error, as well as Eq. (8), is generated as

$$
(R H S)=S_{G}+S_{C}
$$

where the discretization error is treated as the correction term $S_{C}$. If the grid spacing is zero, the correction term is a zero vector. In this study, the gravity term is evaluated as a source term. Moreover, the discretization error of the gravity term and the error caused by the nonphysical wave in the Riemann solver are avoided by evaluating the correction term and setting the threshold value. ${ }^{7,8)}$ The correction term is derived as described in section 3.5. The threshold value is set as fol- 
lows. When the fluctuation component of the velocities $u, v$, and $w$ is less than $10^{-15}$ times the flight velocity, each fluctuation component is treated as zero. Moreover, when the fluctuation component of the quantities $p$ and $\rho$ is less than $10^{-15}$ times the quantity of ISA, each fluctuation component is treated as zero. To decide the threshold value, preliminary calculations were performed, in which the orders of the values differed. As a result, the most appropriate value was set to reduce the errors caused by the nonphysical wave in the Riemann solver.

In the case of stratified atmosphere, the boundary condition at the surface of the body must be set considering the change caused by the stratified atmosphere and the fluctuation caused by the waves in the compressible fluid. However, these two terms cannot be distinguished. Thus, simple extrapolation such as that used in the unstratified atmosphere is not necessarily adequate. To improve this problem, uniform properties of atmosphere were assumed for the nearfield; that is, the region of computation was divided into two zones. In the near-field zone from $r / L=0$ to 1 , axisymmetric Euler analysis was adopted on the assumption of uniform atmospheric properties. However, to reduce the influence of the outer boundary, the computation was performed from $r / L=0$ to 10 , where the extended region was set from $r / L=1$ to 10 . In the far-field zone from $r / L=1$ to $110,3 \mathrm{D}$ Euler equations were used with the stratified atmosphere model. In this 3D analysis, the flow properties from $r / L=0$ to 1 were set by interpolating fluctuation components based on the results of the axi-symmetric Euler analysis. In addition, the properties were used to calculate numerical fluxes near $r / L=1$, although they were not changed in the $3 \mathrm{D}$ analysis.

The inviscid terms were computed using the Simple Highresolution Upwind Scheme (SHUS), ${ }^{12)}$ which is extended to third-order accuracy by the Monotone Upstream-centered Schemes for Conservation Law (MUSCL) interpolation ${ }^{13}$ ) with the van Albada slope limiter. ${ }^{14)}$ The time integration was performed using the first-order Matrix-Free GaussSeidel (MFGS) method. ${ }^{15)}$ In the simulation, a double-precision calculation was performed.

\subsection{Computational grid}

Figure 4 shows an outline of the computational grid in which a two-dimensional grid was rotated about the $x$ axis from $0^{\circ}$ to $180^{\circ}$ to construct a $3 \mathrm{D}$ grid. Because the atmospheric temperature is not constant with altitude, as shown in Fig. 3(a), the shock-wave angles differed according to the altitude. To precisely evaluate the sonic boom intensity, it is essential to capture the shock wave as precisely as possible. In this study, a solution-adapted grid was constructed to align the grid lines to the front and rear shock waves of the incoming waveform in the entire computational domain. The locations of the front and rear shock waves were set as follows. The locations of the highest and second-highest pressure gradients were derived. Then, the derived upstream and downstream locations were treated as the locations of the front and rear shock waves, respectively. At the front and rear shock waves of the incoming waveform, the grid spacing

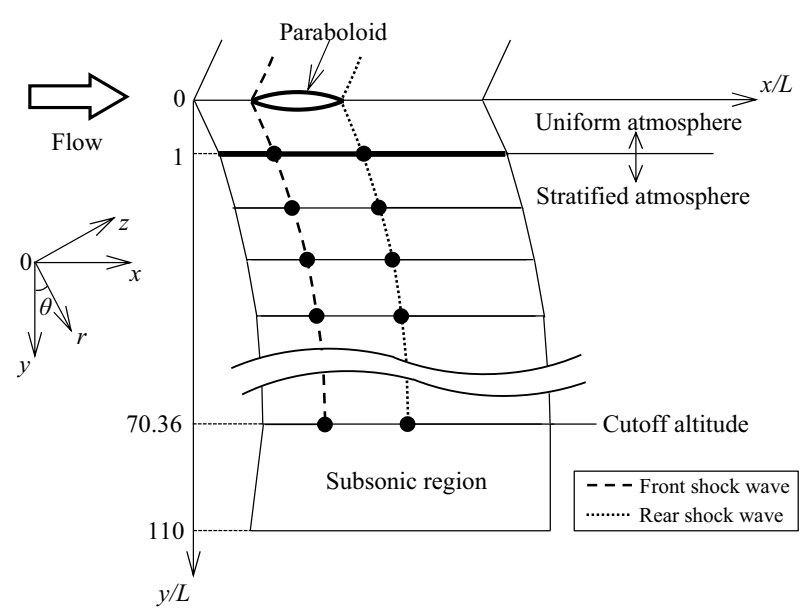

Fig. 4. Outline of the computational grid.

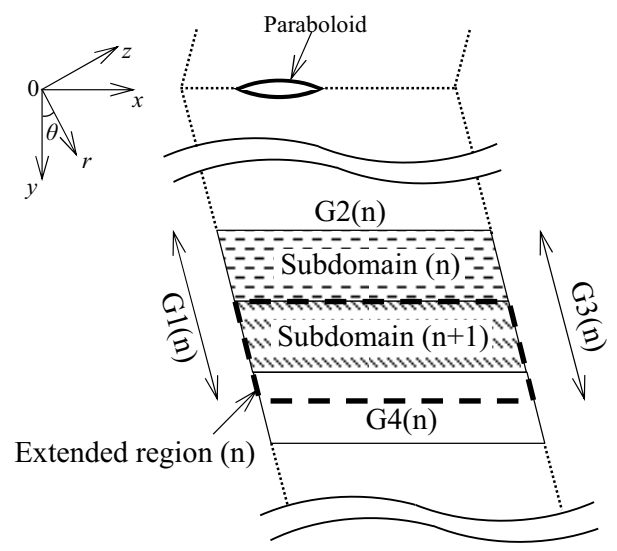

Fig. 5. Outline of subdomain and extended region.

Table 1. Range of subdomain (n) with extended region (n).

\begin{tabular}{lcc}
\hline Atmosphere & $\mathrm{n}$ & $r / L$ \\
\hline Uniform & 0 & $0-10$ \\
\hline Stratification & $1-7$ & $1-5.8,2.2-8, \cdots, 15.6-27$ \\
& $8-22$ & $20-32,25-37, \cdots, 90-102$ \\
& 23 & $95-110$ \\
\hline
\end{tabular}

in the streamwise direction was the minimum.

To reduce the computational cost, the computational domain was split into a sequence of subdomains in the radial direction when the solution-adapted grid was constructed. When the computation in the subdomain (n) was performed, the extended region (n) was computed simultaneously to prevent the influence of the boundary condition at the maximum value of $r$ in the subdomain (n). Figure 5 shows the outline of the subdomain and the extended region, where G1(n)-G4(n) are the boundaries of the subdomain (n) and extended region (n). Table 1 shows the range of each subdomain with the extended region. At $r / L \leq 20$, the grid spacing increased with the radial distance. At $r / L \geq 20$, the grid spacing, nondimensionalized with the body length, was set to 1 . The grid angle was changed every 10 points at $r / L \leq 5$ and every 5 points at $r / L \geq 5$ to make the grid lines smooth. Moreover, the grid angles in each rotational direction ( $\theta$ direction) were 


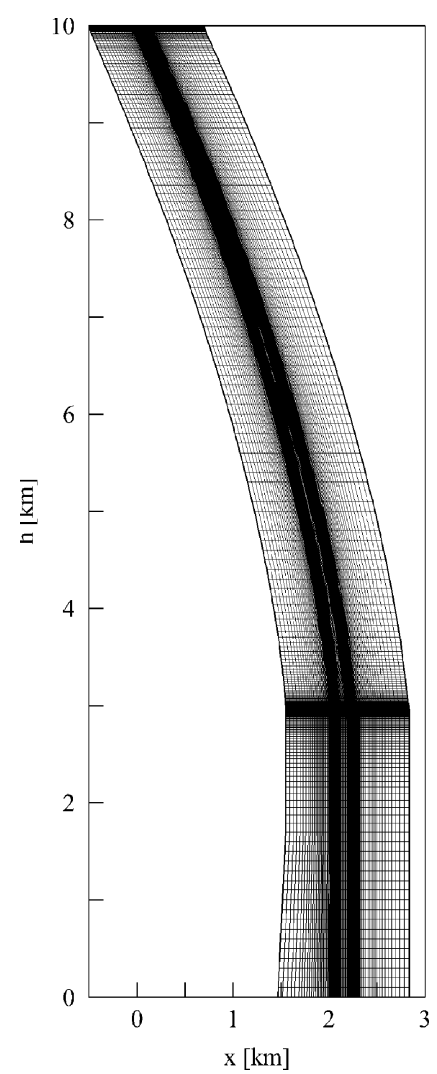

Fig. 6. Computational grid of Grid (3A) in the symmetry plane beneath the body.

Table 2. Number of computational nodes.

\begin{tabular}{llccc}
\hline & & $\begin{array}{c}\text { Streamwise } \\
\text { direction }\end{array}$ & $\begin{array}{c}\text { Radial } \\
\text { direction }\end{array}$ & $\begin{array}{c}\text { Rotational } \\
\text { direction }\end{array}$ \\
\hline 2D (axi- & Grid (2A) & 241 & 87 & - \\
symmetric) & Grid (2B) & 481 & 87 & - \\
& Grid (2C) & 241 & 173 & - \\
\hline 3D Grid & & 241 & 136 & 53 \\
(Grid generation) & & & \\
\hline 3D & Grid (3A) & 241 & 296 & 53 \\
& Grid (3B) & 481 & 296 & 53 \\
& Grid (3C) & 241 & 591 & 53 \\
& Grid (3D) & 241 & 296 & 103 \\
\hline
\end{tabular}

also changed. Grid generation under the above conditions was conducted in the entire computational domain. Thereafter, the grid number in the radial direction was increased in the cutoff region because the flow field around the region is very complex. Specifically, the location of the minimum grid spacing in the radial direction was set at $r / L=70.36$, where the pressure rise reached the local maximum value by sonic cutoff. Figure 6 shows the reconstructed grid at $\theta=0^{\circ}$, which is the symmetry plane beneath the body. Finally, numerical calculation in the far-field zone from $r / L=1$ to 110 was performed using the reconstructed grid.

The number of the computational nodes is shown in Table 2. The grid convergence was confirmed by comparison with results using grids with twice the number of Grid (2A) or Grid (3A). Computational grids except for these two were constructed by adding the internal dividing points

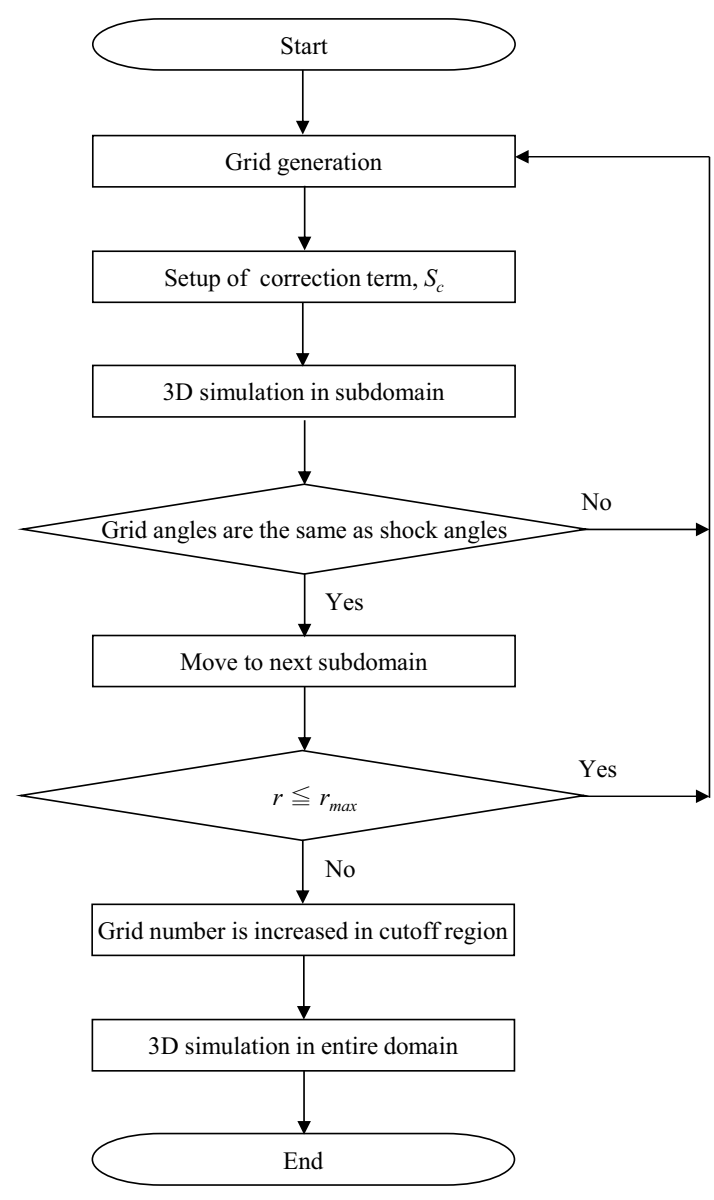

Fig. 7. Flowchart of full-field simulation.

to Grid (2A) or Grid (3A). By comparing the results in Grid (2A) with those in the other two-dimensional grids, the differences in the maximum pressure rise at $r / L=1$ were $0.2 \%$ in Grid (2B) and $-0.3 \%$ in Grid (2C). Moreover, by comparing the results in Grid (3A) with those in the other $3 \mathrm{D}$ grids, the differences in peak pressure rise in the cutoff region were $6.3 \%$ in Grid (3B), $-0.7 \%$ in Grid (3C), and $3.2 \%$ in Grid (3D). These results show that the grid convergences are adequate for evaluating the nature of cutoff phenomena.

\subsection{Computational procedure}

Figure 7 shows a flowchart of the full-field simulation. The computational procedure was divided into two stages. The first is grid generation to adapt the flow field, particularly the shock pattern, and the second is computation of the entire domain using the solution-adapted grid. In the first stage, grid generation was performed as follows.

1. The computational grid was set in the subdomain (n) with the extended region (n) that is surrounded by G1(n)G4(n), as shown in Fig. 5.

2. The correction term in Eq. (9) was set as follows. The free-stream conditions in the stratified atmosphere, where $u=u_{\mathrm{ref}}, v=w=0, T=T_{\infty}(h)$, and $p=p_{\infty}(h)$, were set in the entire domain involving the boundaries G1(n)G4(n). The numerical fluxes were then computed. Next, the correction term was determined to conserve the freestream condition in the stratified atmosphere as follows. 


$$
S_{C, i, j, k}=\left(\frac{\partial E}{\partial x}+\frac{\partial F}{\partial y}+\frac{\partial G}{\partial z}-S_{G}\right)_{i, j, k}
$$

Thereafter, the correction term was evaluated as the constant value.

3. 3D Euler equations with the gravity term were numerically solved. In the case of stratified atmosphere, the atmospheric properties vary with altitude. Therefore, the boundary conditions were set as follows. The flow properties at G2(n) were set as those obtained in the subdomain $(n-1)$ (at $r / L=1$, the properties were set by interpolating the fluctuation components based on the results of the axi-symmetric analysis). G4(n) must be set considering the change caused by atmospheric stratification and the fluctuation caused by waves in the compressible fluid. However, when the boundary condition is set, these two terms cannot be distinguished. Thus, simple extrapolations such as those used in the unstratified atmosphere and nonreflective boundary conditions are not necessarily adequate. In this study, G4(n) was treated as the free-stream condition in the stratified atmosphere. However, the subdomain (n) and extended region (n) were computed simultaneously. The distance of the extended region (n) was sufficiently far to prevent the influence of the boundary condition at G4(n) from reaching the subdomain (n). As a result, the influence of the nonphysical wave generated at G4(n) in the stratified atmosphere was successfully avoided. G1(n) was treated as the free-stream condition in the stratified atmosphere, and G3(n) was treated as the zeroth-order extrapolation in the horizontal direction.

4. The solution-adapted grid construction was validated. If the grid lines were not aligned to the front and rear shock-wave surfaces, computation involving the grid generation was performed again in the same subdomain. Otherwise, the computation was performed in the next subdomain.

Computation in accordance with the above procedure was iterated until grid generation in the entire computational domain was finished. Thereafter, the grid number in the radial direction was increased in the cutoff region. As a result, the solution-adapted grid in the entire computational domain was constructed.

In the second stage, computation in the entire domain from $r / L=1$ to 110 was performed using the solution-adapted grid. Then, the results from $r / L=0$ to 100 were investigated. Consequently, the world's first computation to simulate the $3 \mathrm{D}$ structure of sonic boom cutoff phenomena was successful.

In this study, the computations were performed using the HITACHI SR16000 System (yayoi) in the Information Technology Center, The University of Tokyo. The computational capability was 980.48 GFlops per node. It took approximately 10 hours for solution-adapted grid generation and approximately 24 hours for computation using Grid (3A), when one node was used.

\section{Computational Results}

Pressure rise, which is the most important parameter for validating the numerical accuracy of sonic booms, is evaluated in this chapter. Grid (2B) and Grid (3B) were used for the analysis because the peak pressure rise in the cutoff region obtained for these grids was the highest among the results for all computational grids. The results obtained using Grid (2B) and Grid (3B) are as follows.

\subsection{Pressure distribution}

Figures 8-10 show the distributions of the pressure rise $\Delta p$ in the symmetry plane beneath the body. The sonic line, which indicates the boundary of the supersonic and subsonic regions, is plotted as the white line in these figures. Physical phenomena around the paraboloid include the front shock wave from the nose, the expansion wave from the convex surface of the body, and the rear shock wave from the tail. A large variation in flow field was observed at an altitude of $2.5-4 \mathrm{~km}$, indicating sonic cutoff, which was caused by the diffraction of rays. In the cutoff region, the incoming wave generated from the paraboloid was divided into an outgoing wave in the upward direction and an evanescent wave in the downward direction. Furthermore, the pressure fluctuation of the outgoing wave was confirmed to propagate in the upward direction. Note that the incoming wave appeared to be simply reflected at the cutoff surface. However, this result should be interpreted as diffraction phenomena. As shown in Fig. 10, the shock wave was vertical to the cutoff surface. Unless the angle between the reflection surface and shock

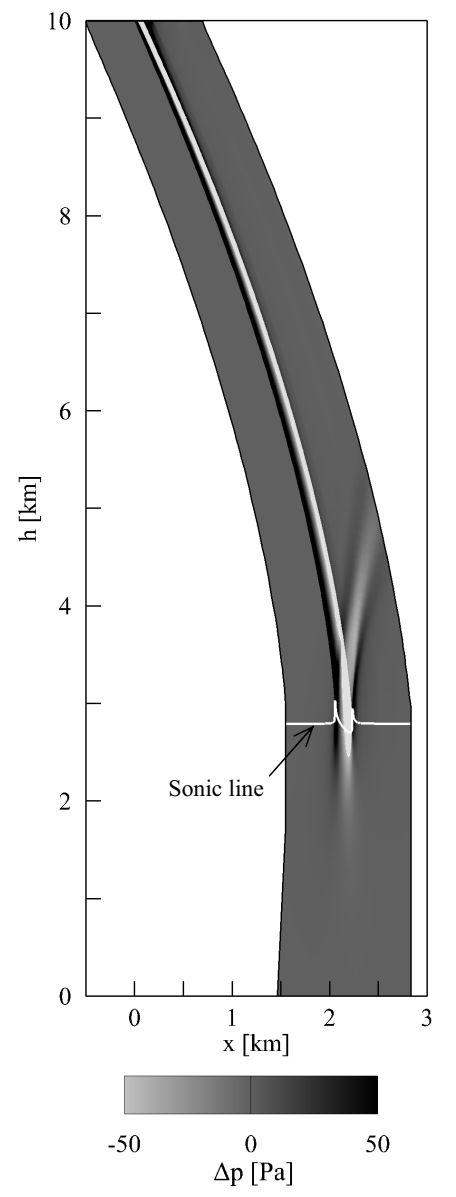

Fig. 8. Overall distribution of pressure rise in the symmetry plane beneath the body. 


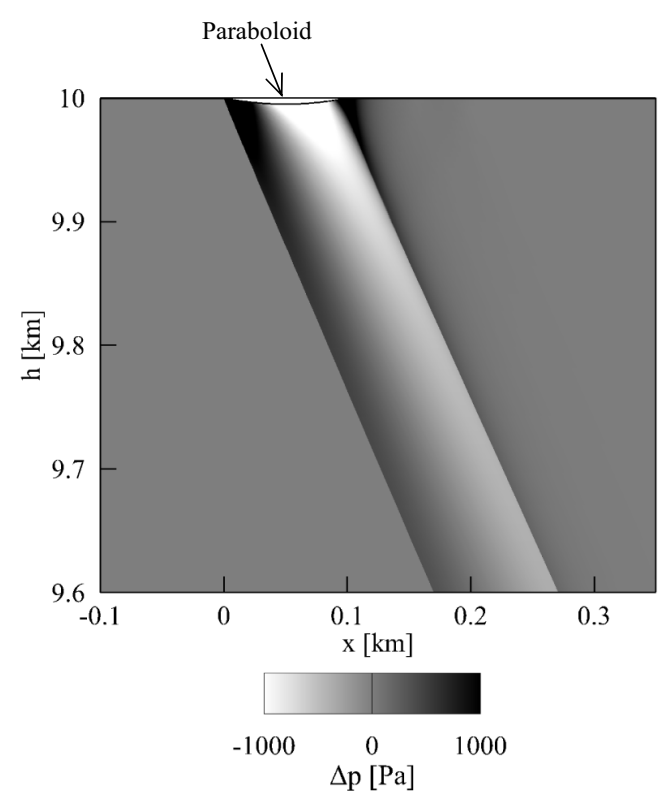

Fig. 9. Pressure rise distribution at near-field in the symmetry plane beneath the body.

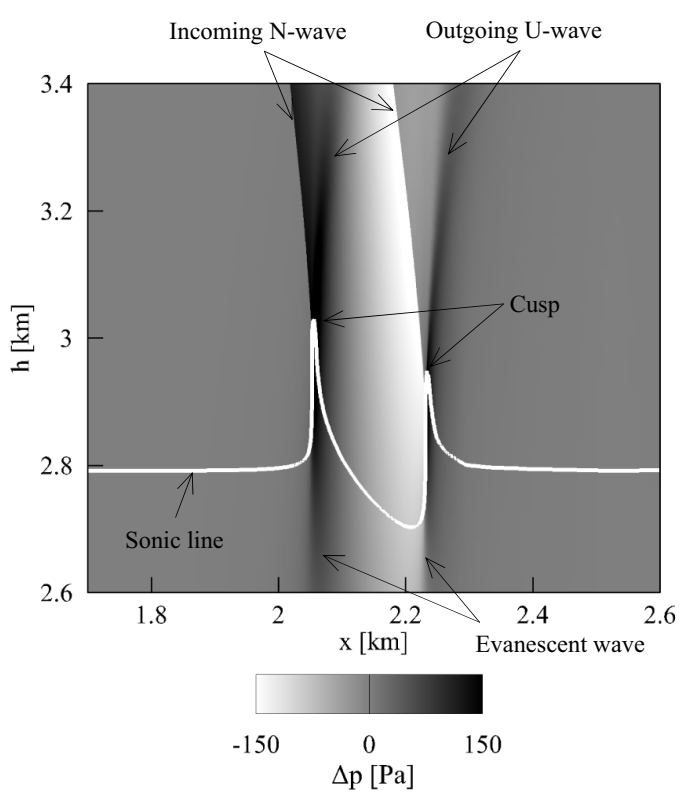

Fig. 10. Pressure rise distribution in the cutoff region in the symmetry plane beneath the body.

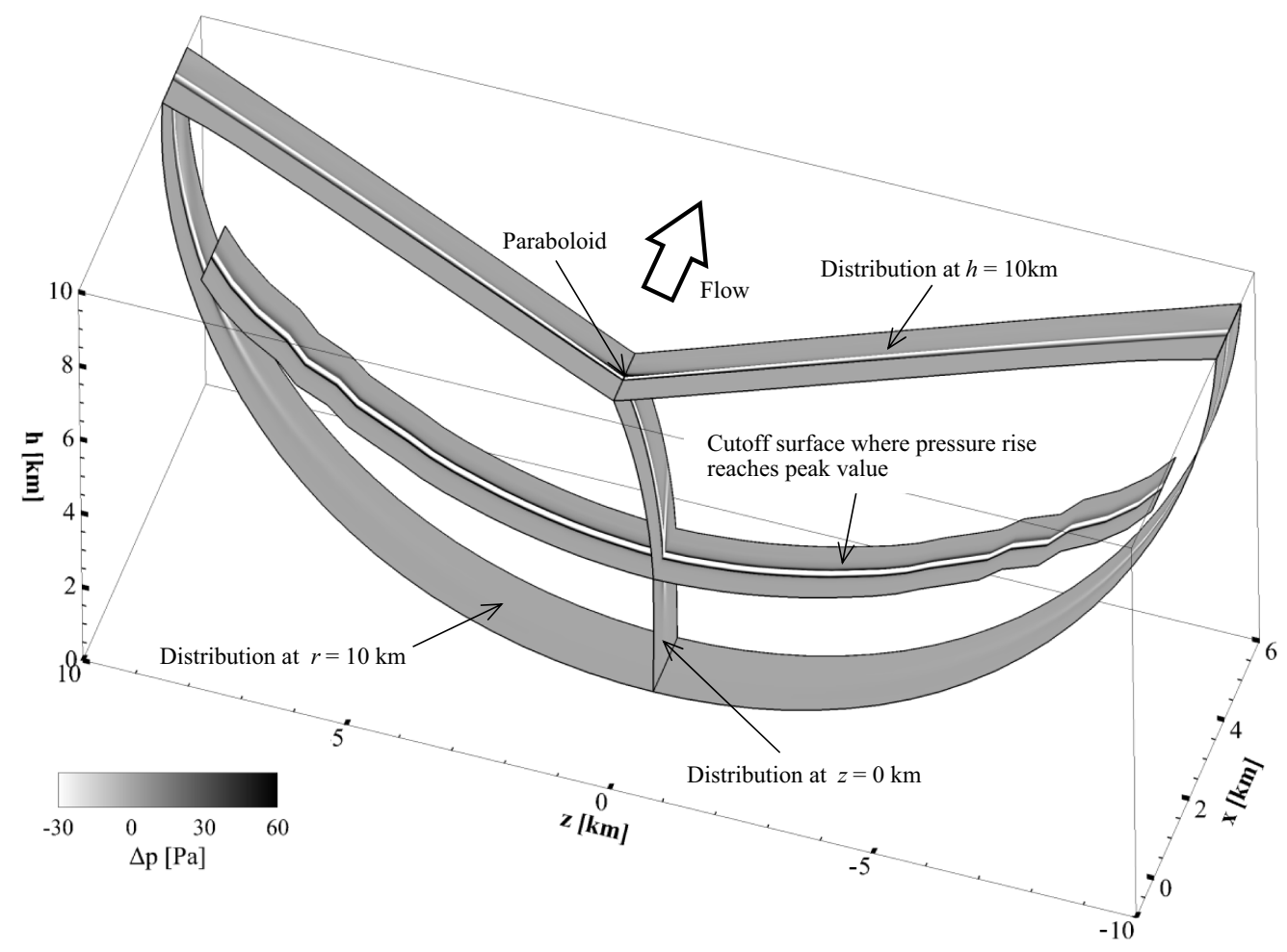

Fig. 11. Three-dimensional distribution of pressure rise involving cutoff surface

wave is less than $90^{\circ}$, reflection never occurs. Therefore, it is reasonable to consider cutoff as a diffraction phenomenon. In addition, the existence of the cusp in the cutoff region corroborates this fact because it cannot appear in the reflection. As previously mentioned, the complicated structure of the flow field can be clarified in the case of full-field simulation; this is a specific advantage of the simulation. The cutoff altitude in which the local Mach number is equal to 1 is $2.79 \mathrm{~km}$ in the uniform flow, and pressure fluctuation increases in the cutoff region. Moreover, the evanescent wave under the cutoff re- gion is reduced rapidly because the compression waves are not converged in the subsonic region. These phenomena are generated when the atmospheric temperature and speed of sound increase toward the ground.

Figure 11 shows the overall distribution of the pressure rise from $\theta=-90^{\circ}$ to $90^{\circ}$. In the figure, the pressure rise distributions are shown at $h=10 \mathrm{~km}, z=0 \mathrm{~km}$, and $r=10 \mathrm{~km}$. Moreover, the distribution in which the pressure rise reaches the peak value in each rotational direction is shown as the cutoff surface. Considering the cutoff surface, the altitude 


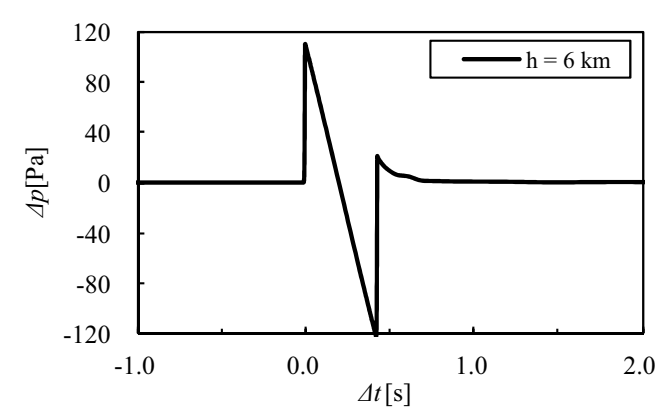

(a) $h=6 \mathrm{~km}$

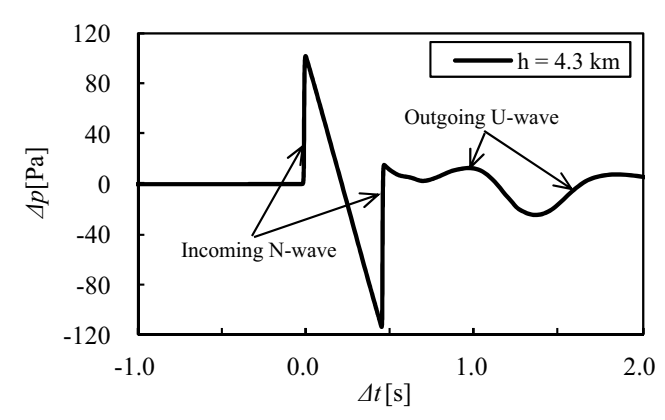

(b) $h=4.3 \mathrm{~km}$

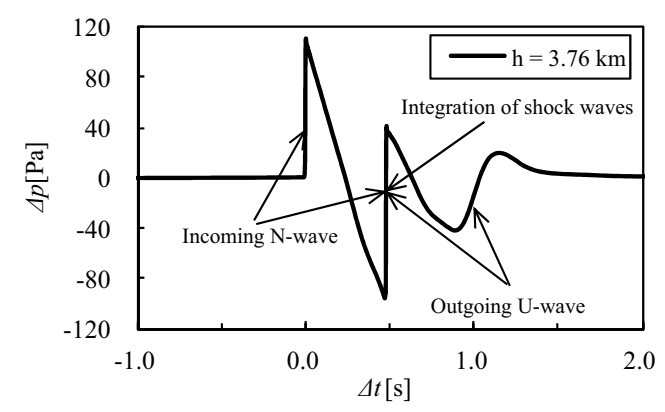

(c) $h=3.76 \mathrm{~km}$

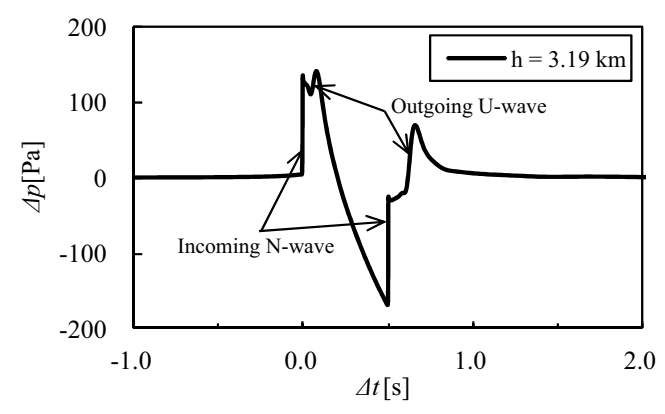

(d) $h=3.19 \mathrm{~km}$

of lateral cutoff is higher than that of sonic cutoff. The shape of the cutoff surface is downward convex. These results demonstrate that the 3D structure of cutoff phenomena can be investigated using computational results.

The analysis of the flight test data of SCAMP and the results obtained using the lossy nonlinear Tricomi equation ${ }^{6)}$ confirmed that the incoming wave is divided into an outgoing wave and an evanescent wave. These results also demonstrate that the outgoing wave is weaker than the incoming wave and that the pressure rise increases in the vicinity of the caustic region. It was also shown that attenuation of the eva-

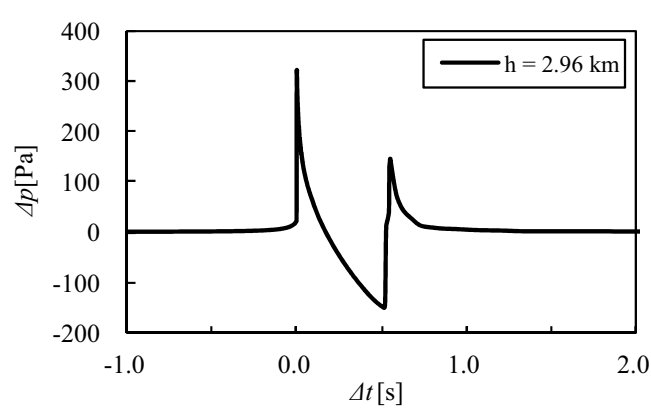

(e) $h=2.96 \mathrm{~km}$

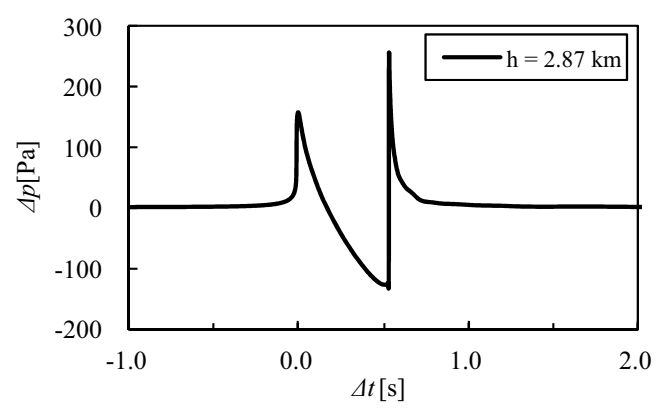

(f) $h=2.87 \mathrm{~km}$

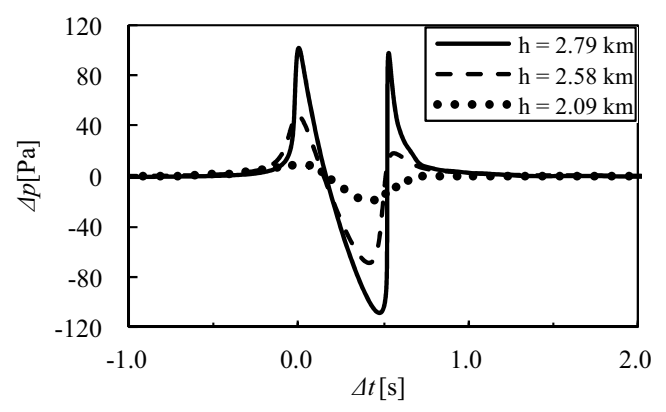

(g) $h=2.79,2.58,2.09 \mathrm{~km}$

Fig. 12. Pressure waveform in the symmetry plane beneath the body.

nescent wave is fast. In general, the characteristics of the pressure waveform predicted by the full-field simulation agreed well with those obtained by the SCAMP flight test and the analysis based on the lossy nonlinear Tricomi equation. Therefore, full-field simulation is readily available and useful for investigating detailed mechanisms of cutoff phenomena.

\subsection{Pressure waveform}

Figure 12 shows the pressure waveforms in the symmetry plane beneath the body. In the figure, the horizontal axis represents the relative time calculated by the streamwise distance from the front shock wave and flight velocity. Note that 
the pressure distributions shown in Figs. 8-11 obtained by full-field simulation are useful for understanding the transition of the pressure waveform toward the ground. As shown in Fig. 12(a), the configuration of the pressure waveform at $h=6 \mathrm{~km}$ is the N-wave. As shown in Fig. 12(b), the incoming waveform upstream and the outgoing waveform downstream are confirmed at $h=4.3 \mathrm{~km}$. Then, the rear shock wave of the incoming waveform interacts with the front shock wave of the outgoing waveform at $h=3.76 \mathrm{~km}$, as shown in Fig. 12(c). As shown in Fig. 12(d), the incoming and outgoing waveforms at $h=3.19 \mathrm{~km}$ are close and overlap. As shown in Fig. 12(e), the pressure rise of the front shock wave exceeds that of the rear shock wave at $h=2.96 \mathrm{~km}$. As shown in Fig. 12(f), the pressure rise of the rear shock wave at $h=2.87 \mathrm{~km}$ reaches the peak value after integration of the rear shock wave of the incoming and outgoing waveforms. Then, Figs. 12(e) and (f) show that sonic boom intensity changes dramatically in the cutoff region. As shown in Fig. 12(g), the pressure waveforms at various altitudes below the cutoff surface indicate that the evanescent wave was rapidly reduced in the downward propagation.

\subsection{Maximum pressure}

Figure 13 shows the distribution of maximum pressure rise of the front shock-wave surface viewed from upstream. The distribution is described from $\theta=0^{\circ}$ to $360^{\circ}$, considering symmetry with respect to the $x-y$ plane. The change in maximum pressure rise around an altitude of $11 \mathrm{~km}$ is influenced by the change in atmospheric temperature gradient, as shown in Fig. 3(a). The attenuation of the sonic boom is different in the direction of propagation. Then, the pressure rise increases dramatically in the caustic region, which has a downward convex shape.

Figure 14 shows the maximum pressure rise of the front shock wave with altitude in the symmetry plane beneath

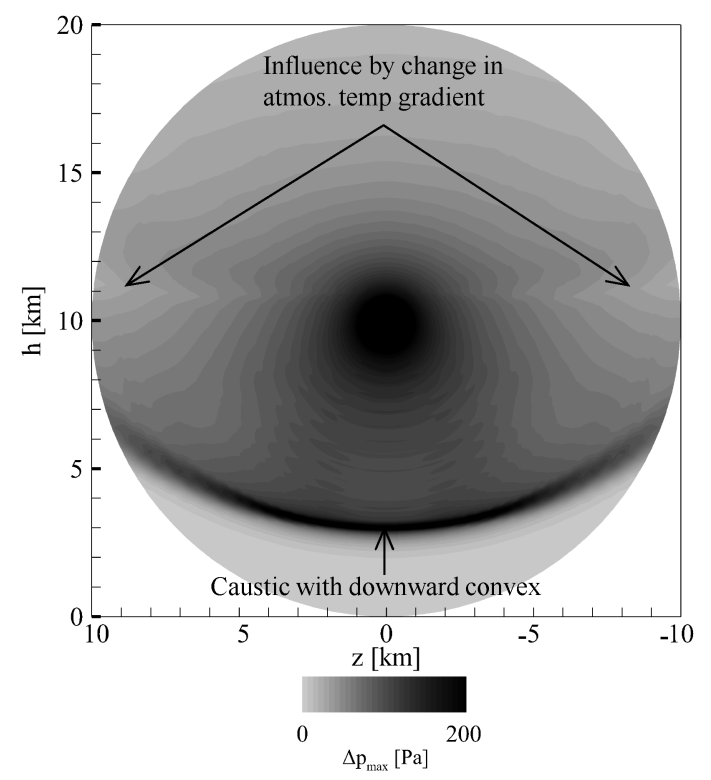

Fig. 13. Maximum pressure rise at front shock-wave surface. the body. Although the correction term and threshold values were set to simulate the flow field in the stratified atmosphere as described in section 3.3, small numerical errors were generated. As shown in the figure, the maximum pressure rise at the near-field decreased rapidly because of the strong effect of geometrical spreading, which indicates the attenuation effect caused by the spreading of the shock wave as distance from the paraboloid increased. The peak pressure rise occurred at $h=2.96 \mathrm{~km}$ in the cutoff region. The peak value of the pressure rise was $319 \mathrm{~Pa}$ and 3.1 times larger than the local minimum value at the upper altitude. This result was obtained because the pressure rise reached the peak value when the focusing of rays caused by the diffraction effect was strongest in the cutoff region. For the evanescent wave under the cutoff altitude, the maximum pressure rise at $h=2.09 \mathrm{~km}$ was approximately $10 \mathrm{~Pa}$ and the tenth part of that at $h=2.79 \mathrm{~km}$, which is the sonic cutoff altitude in the uniform flow. Therefore, the influence of sonic boom can be ignored at the ground when the flight is assumed to have a speed of Mach 1.1 at an altitude of $10 \mathrm{~km}$. Because of the presence of cutoff phenomena, aircraft will be allowed to fly at a relatively low supersonic Mach number, even though the shock waves are formed around the aircraft. Although the allowable Mach number is limited, the flight

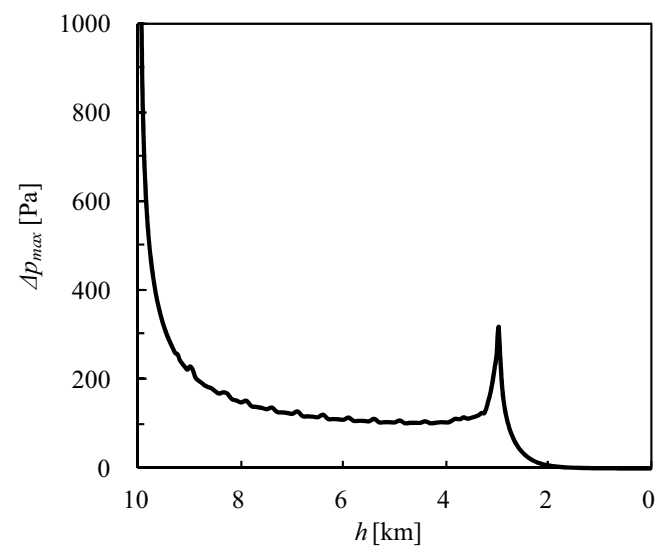

Fig. 14. Maximum pressure rise of front shock wave with altitude in the symmetry plane beneath the body.

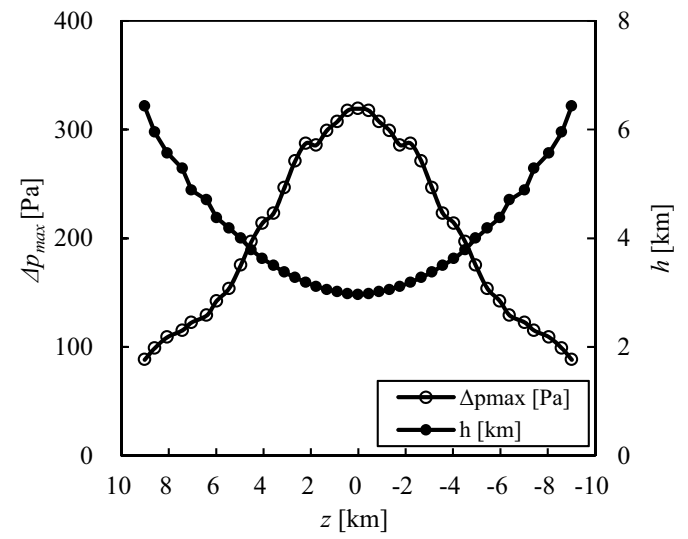

Fig. 15. Peak pressure rise of front shock wave and altitude in sonic and lateral cutoffs. 
velocity of the aircraft is significantly higher than that of present transonic civil aircraft. Then, full-field simulation is useful for determining the appropriate flight conditions.

Figure 15 shows the peak pressure rise and altitude in both sonic and lateral cutoffs, where the pressure rise of the front shock wave reaches the peak value in each rotational direction. Small numerical errors, as well as those shown in Fig. 14, were generated. Moreover, because all peak values were obtained at grid points, they were not necessarily the maximum in the cutoff region. For this reason, the curve of the peak pressure rise is not smooth. In Fig. 15, the peak pressure rise in the lateral cutoff becomes low, and the lateral cutoff altitude becomes high as the distance from the $x-y$ plane increases.

\section{Conclusions}

In this study, we successfully performed the first ever direct simulation of cutoff phenomena. The results show that sonic cutoff occurs when the local Mach number is 1 , and that the incoming wave generated from the paraboloid is divided into an outgoing wave in the upward direction and an evanescent wave in the downward direction at the cutoff altitude. Moreover, sonic boom is enhanced by the cutoff phenomena in the cutoff region where peak pressure rise is increased. For the evanescent wave, the maximum pressure rise attenuates rapidly under the cutoff altitude. Therefore, the influence of sonic boom can be ignored at the ground when a supersonic aircraft flies at a relatively low supersonic speed. When compared to sonic cutoff, lateral cutoff occurs at a higher altitude, and the shape of the cutoff surface is downward convex. Moreover, it is shown that the peak pressure rise in lateral cutoff becomes low as the distance from the vertical plane including the flight path increases.

\section{References}

1) Aerion AS2, http://www.aerionsupersonic.com/

2) Page, J. A., Plotkin, K. J., Haering, E. A., Jr., Maglieri, D. J., Cowart, R., Salamone, J., Elmer, K., Welge, B., and Ladd, J.: SCAMP: Superboom Caustic Analysis and Measurement Project Overview, AIAA Paper 2013-0930, 2013.

3) NASA FaINT Project, http://www.nasa.gov/topics/aeronautics/features/ faint_sonic_booms.html

4) McDonald, B. E.: High-angle Formulation for the Nonlinear Progressive-wave Equation Model, Wave Motion, 31 (2000), pp. 165-171.

5) Auger, T. and Coulouvrat, F.: Numerical Simulation of Sonic Boom Focusing, AIAA J, 40 (2002), pp. 1726-1734.

6) Salamone, J. A., III, Sparrow, V. W., and Plotkin, K. J.: Solution of the Lossy Nonlinear Tricomi Equation Applied to Sonic Boom Focusing, AIAA J., 51 (2013), pp. 1745-1754.

7) Yamashita, R. and Suzuki, K.: Sonic Boom Analysis for Hypersonic Vehicle by Global Direct Simulation, J. Jpn. Soc. Aeronaut. Space Sci., 62 (2014), pp. 77-84 (in Japanese).

8) Yamashita, R. and Suzuki, K.: Full-Field Sonic Boom Simulation in Real Atmosphere, AIAA Paper 2014-2269, 2014.

9) Thomas, C. L.: Extrapolation of Sonic Boom Pressure Signatures by the Waveform Parameter Method, NASA TN D-6832, 1972.

10) D-SEND Database, http://d-send.jaxa.jp/d_send_e/index.html [cited May 30, 2012].

11) ISO 2533, 1975

12) Shima, E. and Jounouchi, T.: Role of CFD in Aeronautical Engineering (No. 14)_AUSM Type Upwind Schemes-, NAL SP-34, 1997, pp. 7-12.

13) van Leer, B.: Towards the Ultimate Conservative Difference Scheme. IV. A New Approach to Numerical Convection, J. Comput. Phys., 23 (1977), pp. 276-299.

14) van Albada, G. D., van Leer, B., and Roberts, W. W., Jr.: A Comparative Study of Computational Methods in Cosmic Gas Dynamics, Astronomy and Astrophysics, 108 (1982), pp. 76-84.

15) Shima, E.: A Simple Implicit Scheme for Structured/Unstructured CFD, Proceedings of 29th Fluid Dynamic Conference, 1997, pp. 325-328 (in Japanese).

$\mathrm{S} . \mathrm{Fu}$

Associate Editor 